\title{
Optical characterization of tissue-simulating phantoms with microparticles by Digital Image Plane Holography
}

\author{
Laura Arévalo-Díaz, Félix Fanjul-Vélez*, Miguel A. Rodríguez-Colmenares, José L. Arce-Diego* \\ Applied Optical Techniques Group, TEISA Department, \\ University of Cantabria, Av. de los Castros s/n, 39005, Santander (Spain)
}

\begin{abstract}
Digital Image Plane Holography (DIPH) is a non-invasive optical technique which is able to recover the whole object wave. An object is illuminated and the diffused backscattered light is carried to a digital sensor by using a lens, where it interferes with a divergent reference wave with its origin in the lens aperture plane. Selecting each aperture image in the Fourier plane, the amplitude and the phase of the object beam are obtained. If two holograms are recorded at different times, after a small displacement, the reconstructed intensity distributions can be taken as a speckle field, while the phase difference distribution can be analyzed by an interferometric approach. In this work scattering media are investigated by using digital holography. The aim of this paper is to determine the viability of the technique to characterized optical properties of the sample. Different scattering media are modeled with different scattering properties. Each model generates a speckle pattern with different statistical properties (size, contrast, intensity). Both the visibility of the interferometric fringes and the properties of speckle pattern are related with optical properties of the media such as absorption and scattering coefficient. The ability to measure these properties makes the technique a promising method for biomedical applications.
\end{abstract}

Keywords: Digital holography, scattering media, speckle pattern, optical properties, fringe visibility.

\section{INTRODUCTION}

There is an increasing interest in the study of diffusing media by using optical techniques. Digital Image Plane Holography (DIPH) is a non-intrusive, non-contact and high sensitivity optical technique. A numerical reconstruction allows to retrieve the original object wavefront. The technique can resolve optical path changes up to one hundredth of a wavelength [1]. To record a digital hologram, two wavefronts (object and reference beam) interfere in the digital sensor. In the set-up, an appropriate angle between the reference and the object beams is required, in this way the amplitude and the phase of the object beam is recovered from a single record. In this work, we propose to use both the amplitude and phase information to study scattering samples. The objective is to determine the viability of the technique to characterize samples with different optical properties.

For scattering samples, the amplitude recovered will be a speckle pattern. A coherent illumination produces a speckle pattern due to the interference of light scattered by the object. Statistical properties of speckle fields are well-known [2]. Speckle analysis has been used to characterize the dynamic behavior of samples with biological activity $[3,4]$. In the case of a turbid media, speckle pattern parameters are related with the optical properties of the media, as scatterer sizes, absorption coefficient or refractive index [5,6]. This relation can be used to infer information about the sample from the backscattered speckle. On the other hand, if the sample has moved and two holograms have been recorded, a phase difference map is obtained. Fringes in the phase map are related with the object displacement. In this work, we are interested in the quality of the fringes. This is not the first time that the fringe visibility is useful to characterize surfaces. In [7], the contrast of fringes obtained in an optical interferometer are used to estimate surface roughness. Changes on the interferometric fringe visibility due to microspheres of different sizes has been evaluated [8]. In [9], the relation between fringe visibility and speckle size is described. The contrast of the phase map is used to examine the sample. This paper is organized as follow. Section 2 describes the fundamentals of the Digital Holography. The numerical model, the results and the discussion are included in Section 3. Finally, Section 4 contains the most relevant conclusions.

Quantitative Phase Imaging III, edited by Gabriel Popescu, YongKeun Park, Proc. of SPIE Vol. 10074, 100741R · @ 2017 SPIE · CCC code: 1605-7422/17/\$18 · doi: 10.1117/12.2252597 


\section{DIGITAL HOLOGRAPHY}

Digital holography is a non-invasive optical technique which is able to recover the whole object wave. To record a digital hologram, an object is illuminated and the light scattered by the surface (object wave $o(x, y)$ ) interferes with a reference wave $(r(x, y))$ on a digital sensor, as shown in Figure 1a. These waves have a complex form that can be written as:

$$
o(x, y)=A_{o}(x, y) \exp \left[j \varphi_{o}\right], \quad r(x, y)=A_{r}(x, y) \exp \left[j \varphi_{r}\right],
$$

where $A$ is the real amplitude and $\varphi$ the phase. One of the usual ways to write the hologram intensity is:

$$
I(x, y)=A_{o}^{2}+A_{r}^{2}+2 A_{o} A_{r} \cos \left(\varphi_{o}-\varphi_{r}\right),
$$

where it is clear that the hologram carries information about the phase and the amplitude of the object wave. Since the reference beam is set as a smooth wave, $A_{r}$ is almost constant and $\varphi_{r}$ has a continuous spatial change in the sensor plane, while $A_{r}$ and $\varphi_{r}$ are random variables.
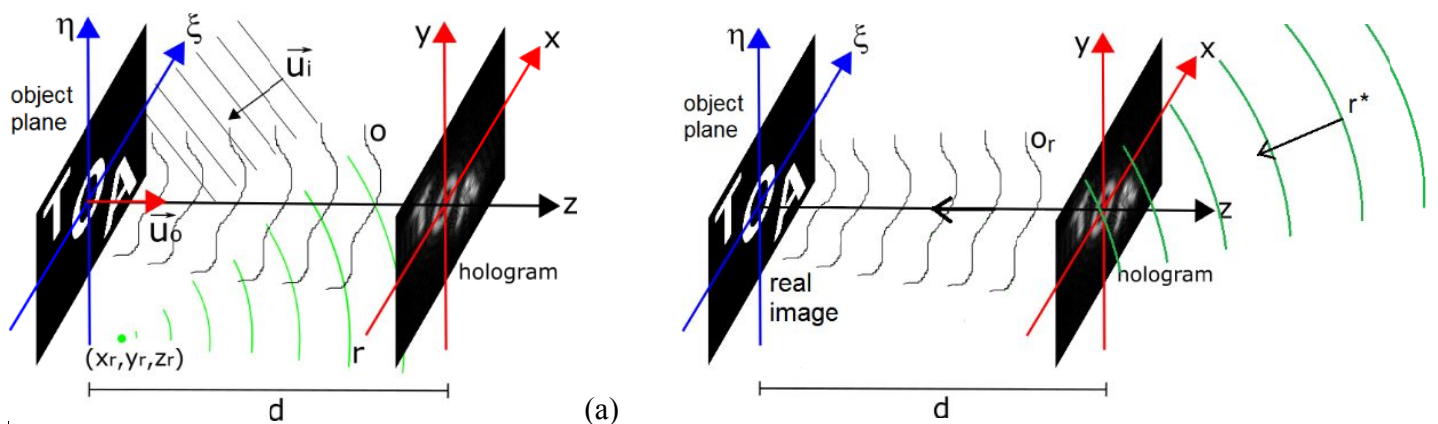

(a)

Figure 1. (a) Hologram recording process. (b) Object wave reconstruction process by using a reconstruction wave $r^{*}$.

For the numerical reconstruction of the complex object wave, the Fresnel-Kirchoff diffraction theory is used [1]. For any plane located in $z$ (Fresnel region: $\xi, \eta \ll z$ ), the reconstructed wave is obtained through a numerical propagation of the hologram $I(x, y)$ multiplied by a reconstruction wave $c(x, y)$ (Figure 1b). Fresnel transformation is defined as:

$$
\Gamma(v, \mu)=\frac{\exp (j k z)}{j \lambda z} \exp \left[j \pi \lambda z\left(v^{2}+\mu^{2}\right)\right] \times \mathfrak{J}^{-1}\left\{I(x, y) c(x, y) \exp \left[\frac{j k}{2 z}\left(x^{2}+y^{2}\right)\right]\right\},
$$

with $\lambda$ the wavelength of the light, $k=2 \pi / \lambda$ the wave number, $v=\frac{\xi}{\lambda z}$ and $\mu=\frac{\eta}{\lambda z}$ scale factors, and $\mathfrak{I}^{-1}$ refers to the inverse Fourier transform. The intensity and the phase are calculated by:

$$
\begin{aligned}
& I(\xi, \eta)=|\Gamma(\xi, \eta)|^{2}, \\
& \varphi(\xi, \eta)=\arctan \frac{\operatorname{Im}[\Gamma(\xi, \eta)]}{\operatorname{Re}[\Gamma(\xi, \eta)]} .
\end{aligned}
$$

In the particular case where a spherical reference beam has its origin in the object plane, the hologram is a lens-less Fourier hologram. Then equation (3) is simplified and the object can be reconstructed by a Fourier transform of the hologram. If the hologram intensity is written as:

$$
I(x, y)=|r(x, y)|^{2}+|o(x, y)|^{2}+r^{*}(x, y) o(x, y)+r(x, y) o^{*}(x, y) .
$$

The Fourier transform of the hologram is given by:

$$
\mathfrak{I}\{I(x, y)\}=\tilde{I}(\xi, \eta)=A_{r}^{2} \delta(0,0)+A_{o}^{2} S(\xi, \eta)+\mathfrak{I}\left\{r^{*} o\right\}+\mathfrak{J}\left\{r o^{*}\right\} .
$$

The two first terms correspond to the reference and object wave spectrum, and lie in the image center. The third and the fourth terms are the virtual and the real images of the object respectively, which are completely separated if an off-axis geometry is used in the setup. 


\subsection{Intensity analysis}

The reconstructed intensity distributions can be taken as a speckle pattern. For an objective speckle formation, the mean speckle size $d_{s}$ depend on the diameter of the illumination area $D$, the wavelength $\lambda$ and the distance between the object and the observation plane $d$, according to the following relation:

$$
d_{s}=1.22 \frac{\lambda d}{D} .
$$

In order to measure the mean speckle size from the intensity distribution, the normalized auto-covariance function of the intensity speckle pattern is required. This function is the same to the normalized autocorrelation function of the intensity. According with the Wiener-Khintchine theorem, the autocorrelation function is given by the Inverse Fourier Transform of the Power Spectral Density of the intensity [2]. Therefore the speckle size is calculated from the spatial intensity distribution by:

$$
c(x, y)=\frac{\mathfrak{J}^{-1}\left\{|\Im\{I(x, y)\}|^{2}\right\}-\langle I(x, y)\rangle^{2}}{\left\langle I(x, y)^{2}\right\rangle-\langle I(x, y)\rangle^{2}} .
$$

The speckle size is estimated of the full width at half-maximum of the autocorrelation function.

\subsection{Phase analysis}

When two holograms are recorded in two different times, separated $\Delta T$, the phase distributions can be analyzed by an interferometric approach [10]. If the object has undergone a displacement $\vec{L}$, the difference between the two phase distributions is related to the displacement such that:

$$
\Delta \phi=\vec{K} \cdot \vec{L},
$$

$\vec{K}$ is called sensitivity vector and gives the direction in which the set-up has maximum sensitivity. The sensitivity vector is defined by the geometry of the experimental setup:

$$
\vec{K}=\frac{2 \pi}{\lambda}\left(\overrightarrow{u_{o}}-\overrightarrow{u_{l}}\right),
$$

where $\overrightarrow{u_{o}}$ y $\overrightarrow{u_{l}}$ are the illumination and observation vectors respectively (Fig. 1). An out-of-plane deformation is measured when illumination and observation vectors are both parallel to $z$. A phase variation of $2 \pi$ corresponds to a deformation of $\lambda / 2$.

In order to evaluate the fringe visibility, the classic definition of fringe visibility is used [11]:

$$
\mathcal{V}=\frac{I_{\max }-I_{\min }}{I_{\max }+I_{\min }},
$$

where $I_{\max }$ and $I_{\min }$ are maximum and minimum intensity in the fringe patter respectively.

\section{NUMERICAL MODEL: RESULTS AND DISCUSSION}

To model the problem, the object plane $(\xi, \eta)$ (see Fig. 1) is sampled on a rectangular matrix of $N \times N$ points, with steps $\Delta$ along the coordinates. The parameters of the numerical model are: $\lambda=630 \mathrm{~nm}, d=1 \mathrm{~m}, N=500, \Delta=0.03 \mathrm{~mm}$. Let us suppose that the object is illuminated by a Gaussian beam with $\overrightarrow{u_{l}}=(0,0,1)$. The intensity distribution on the object surface is show in Figure 2a-b, for beams with different radii $w_{0}$. Different optical properties of the media produce different outgoing diameter scattered light [12]. Using Fresnel-Kirchhoff diffraction integral (equation 3), the speckle pattern intensity in the hologram plane $(x, y)$ can be calculated. It can be seen in Figure 2c-d the speckle field produced for light distribution showed in Figure 2a-c respectively. The mean speckle size of each distribution is different, as can be seen in the figure. 


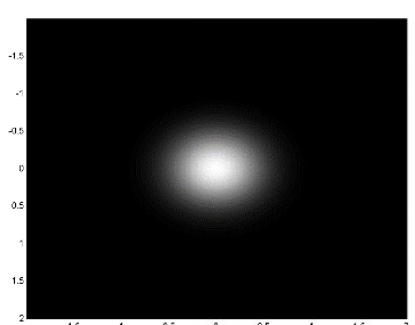

(a)

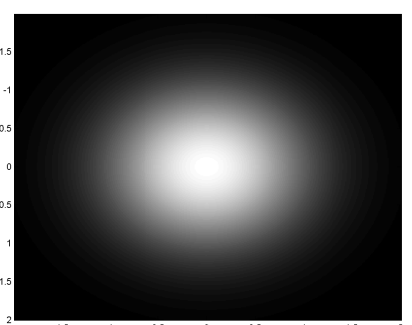

(b)



(c)

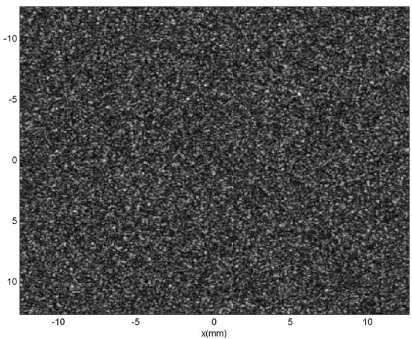

(d)

Figure 2. Intensity of a Gaussian beam in the object plane with (a) $w_{0}=0.45 \mathrm{~mm}$, and (b) $w_{0}=1 \mathrm{~mm}$. Speckle pattern intensity in observation plane with a mean speckle size of (c) $d_{s}=176.5 \mu \mathrm{m}$, and (d) $d_{s}=79.9 \mu \mathrm{m}$.

In Fresnel region, the phase of the divergent reference beam can be modeled as:

$$
\varphi_{r}(x, y) \approx \frac{1}{z_{r}} \exp \left[-i \frac{2 \pi}{\lambda} z_{r}\right] \exp \left[-i \frac{\pi}{\lambda z_{r}}\left\{\left(x-x_{r}\right)^{2}+\left(y-y_{r}\right)^{2}\right\}\right]
$$

with $\left(x_{r}, y_{r}, z_{r}\right)=(4 m m, 0, d)$. This wavefront interferes with object wave (Figure 2c-d) to produce the hologram.

To find a phase difference map, the object is rotated around $\xi$ axis at a given angle of $0.003^{\circ}$, The resulting phase map will be horizontal fringes, where each fringe corresponds to an out-of-plane displacement of $\frac{\lambda}{2}=315 \mathrm{~nm}$. Figure 3 depicts a vertical profile of the phase map, it represents one fringe. Noise curves are due to speckle phenomena. It is clear that the contrast of each map is different when the speckle size is changed. The visibility of the fringes is increased when the speckle size is increased, for a given displacement.

With these results, it can be seen that it is possible to measure characteristics of the object from the quality of the phase map.

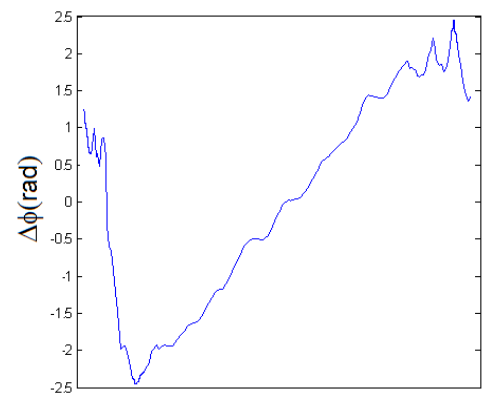

(a)

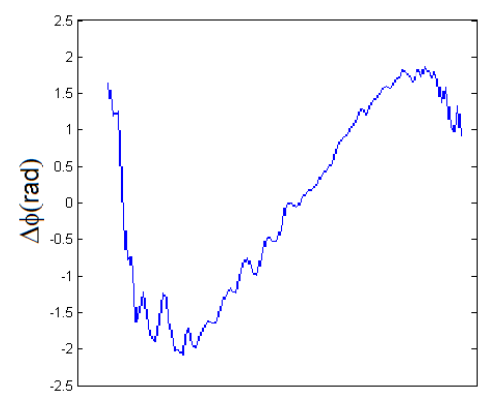

(b)

Figure 3. Phase map intensity profile from a hologram of a speckle pattern with mean speckle size of (a) $d_{s}=172.3 \mu m, v=0.7958$. (b) $d_{s}=57.5 \mu m, \mathcal{V}=0.6366$.

\section{CONCLUSION}

In this preliminary work, we have seen that digital image plane holography is a promising technique to study optical turbid media. The sample is studied in a reflection geometry. Holography allows to recover an intensity and phase distributions of the backscattered light. The mean speckle size of the intensity distribution and the contrast of the phase map are related with optical properties of the media. This makes it possible to infer information about the sample under study. As a future work, we expect to model the backscattered light of a samples with different optical properties by using a Monte Carlo program, in order to find a relation between fringe visibility and absorption or scattering coefficients. 


\section{ACKNOWLEDGEMENTS}

This work has been partially supported by the project "New active phases in transition metals and rare earth nano-oxides stabilized at high pressure" (MAT2015-69508-P) of the Spanish Ministry of Economy and Competitiveness, cofunded by FEDER funds, and by the San Cándido Foundation.

\section{REFERENCES}

[1] Schnars U. and Jueptner W., [Digital Holography], Springer Berlin Heidelberg, New York (2005).

[2] Goodman, J. W., "Statistical properties of laser speckle patterns," Laser speckle and related phenomena. Springer Berlin Heidelberg, 9-75 (1975).

[3] Arizaga, R., Luc1, N., \& Trivi, M., "Display of local activity using dynamical speckle patterns," Optical Engineering 41(2), 287-294 (2002).

[4] Chicea, D., "Biospeckle size and contrast measurement application in particle sizing and concentration assessment," Romanian Journal of Physics 52(5-6), 581-587 (2007).

[5] Piederrière, Y., Cariou, J., Guern, Y., Le Jeune, B., Le Brun, G., and Lortrian, J., "Scattering through fluids: speckle size measurement and Monte Carlo simulations close to and into the multiple scattering," Optics Express 12(1), 176-188 (2004).

[6] Nader, C. A., Nassif, R., Pellen, F., Le Jeune, B., Le Brun, G., \& Abboud, M., "Influence of size, proportion, and absorption coefficient of spherical scatterers on the degree of light polarization and the grain size of speckle pattern," Applied Optics 54(35), 10369-10375 (2015).

[7] Atkinson, J. T., \& Lalor, M. J., "The effect of surface roughness on fringe visibility in optical interferometry," Optics and Lasers in Engineering 1(2), 131-146 (1980).

[8] Martínez-Celorio, R. A., Dávila, A., Pérez-López, C., \& López, L. M., "Visibility enhancement of carrier fringes in Electronic Speckle Shearing Pattern Interferometry using microspheres for light detection in back reflection," Optik-International Journal for Light and Electron Optics 112(3), 99-104 (2001).

[9] Tanner, L. H., "A study of fringe clarity in laser interferometry and holography," Journal of Physics E: Scientific Instruments 1(5), 517 (1968).

[10] Vest C.M., "Holographic Interferometry," Wiley Series in Pure and Applied Optics, Wiley, New York (1979).

[11]Born M, Wolf E: "Principles of Optics". Macmillan, New York (1964).

[12] Piederrière, Y., Boulvert, F., Cariou, J., Le Jeune, B., Guern, Y., \& Le Brun, G., "Backscattered speckle size as a function of polarization: influence of particle-size and-concentration," Optics express 13(13), 5030-5039 (2005). 